$\mathrm{J}$ o u r n a l of

Mathematics

and Applications

JMA No 41, pp 39-51 (2018)

\title{
Nonlinear Fractional Differential Equations with Non-Instantaneous Impulses in Banach Spaces
}

\author{
Mouffak Benchohra and Mehdi Slimane
}

\begin{abstract}
This paper is devoted to study the existence of solutions for a class of initial value problems for non-instantaneous impulsive fractional differential equations involving the Caputo fractional derivative in a Banach space. The arguments are based upon Mönch's fixed point theorem and the technique of measures of noncompactness.
\end{abstract}

AMS Subject Classification: 26A33, 34A37, 34G20.

Keywords and Phrases: Initial value problem; Impulses; Caputo fractional derivative; Measure of noncompactness; Fixed point; Banach space.

\section{Introduction}

The theory of fractional differential equations is an important branch of differential equation theory, which has an extensive physical, chemical, biological, and engineering background, and hence has been emerging as an important area of investigation in the last few decades; see the monographs of Abbas et al. [3, 4], Kilbas et al. [18], Podlubny [23], and Zhou [25], and the references therein.

On the other hand, the theory of impulsive differential equations has undergone rapid development over the years and played a very important role in modern applied mathematical models of real processes rising in phenomena studied in physics, population dynamics, chemical technology, biotechnology and economics; see for instance the monographs by Bainov and Simeonov [12], Benchohra et al. [13], Lakshmikantham et al. [19], and Samoilenko and Perestyuk [24] and references therein. Moreover, 
impulsive differential equations present a natural framework for mathematical modeling of several real-world problems. In pharmacotherapy, instantaneous impulses cannot describe the dynamics of certain evolution processes. For example, when one considers the hemodynamic equilibrium of a person, the introduction of the drugs in the bloodstream and the consequent absorption for the body are a gradual and continuous process. In $[1,2,5,16,22]$ the authors studied some new classes of abstract impulsive differential equations with not instantaneous impulses.

However, the theory for fractional differential equations in Banach spaces has yet been sufficiently developed. Recently, Benchohra et al. [14] applied the measure of noncompactness to a class of Caputo fractional differential equations of order $r \in(0,1]$ in a Banach space. Let $E$ be a Banach space with norm $\|\cdot\|$.

In this paper, we study the following initial value problem (IVP for short), for fractional order differential equations

$$
\begin{gathered}
{ }^{c} D^{r} y(t)=f(t, y(t)), \text { for a.e. } t \in\left(s_{k}, t_{k+1}\right], k=0, \ldots, m, 0<r \leq 1, \\
y(t)=g_{k}(t, y(t)), t \in\left(t_{k}, s_{k}\right], k=1, \ldots, m, \\
y(0)=y_{0},
\end{gathered}
$$

where ${ }^{c} D^{r}$ is the Caputo fractional derivative, $f: J \times E \rightarrow E, g_{k}:\left(t_{k}, s_{k}\right] \times E \rightarrow E$, $k=1, \ldots, m$, are given functions, $J=[0, T]$ and $y_{0} \in E, 0=s_{0}<t_{1}<s_{1}<\cdots<$ $t_{m}<s_{m}<t_{m+1}=T$.

To our knowledge no paper has been considered for non-instantaneous impulsive fractional differential equations in abstract spaces. This paper fills the gap in the literature. To investigate the existence of solutions of the problem above, we use Mönch's fixed point theorem combined with the technique of measures of noncompactness, which is an important method for seeking solutions of differential equations. See Akhmerov et al. [7], Alvàrez [8], Banaś et al. [9, 10, 11], Guo et al. [15], Mönch [20], Mönch and Von Harten [21].

\section{Preliminaries}

In this section, we first state the following definitions, lemmas and some notation. By $C(J, E)$ we denote the Banach space of all continuous functions from $J$ into $E$ with the norm

$$
\|y\|_{\infty}=\sup \{\|y(t)\|: t \in J\} .
$$

Let $L^{1}(J, E)$ be the Banach space of measurable functions $y: J \rightarrow E$ which are Bochner integrable, equipped with the norm

$$
\|y\|_{L^{1}}=\int_{0}^{T}\|y(t)\| d t .
$$




$$
\begin{aligned}
P C(J, E)=\{y: J \rightarrow E: \quad & y \in C\left(\left(t_{k}, t_{k+1}\right], E\right), k=0, \ldots, m \text { and there exist } y\left(t_{k}^{-}\right) \\
& \text {and } \left.y\left(t_{k}^{+}\right), k=1, \ldots, m \text { with } y\left(t_{k}^{-}\right)=y\left(t_{k}\right)\right\} .
\end{aligned}
$$

$P C(J, E)$ is a Banach space with the norm

$$
\|y\|_{P C}=\sup _{t \in J}\|y(t)\|
$$

Set

$$
J^{\prime}=J \backslash \cup_{k=1}^{m}\left(t_{k}, s_{k}\right] .
$$

Moreover, for a given set $\mathrm{V}$ of functions $v: J \rightarrow E$, let us denote by

$$
V(t)=\{v(t), v \in V\}, t \in J
$$

and

$$
V(J)=\{v(t), v \in V, t \in J\} .
$$

Now let us recall some fundamental facts of the notion of Kuratowski measure of noncompactness.

Definition 2.1. ([9]). Let $X$ be a Banach space and $\Omega_{X}$ the bounded subsets of $X$. The Kuratowski measure of noncompactness is the map $\alpha: \Omega_{X} \rightarrow[0, \infty]$ defined by

$$
\alpha(B)=\inf \left\{\epsilon>0: B \subseteq \cup_{i=1}^{n} B_{i} \text { and } \operatorname{diam}\left(B_{i}\right) \leq \epsilon\right\} ; \text { here } B \in \Omega_{X} .
$$

Properties: The Kuratowski measure of noncompactness satisfies the following properties (for more details see [9])

(a) $\alpha(B)=0 \Leftrightarrow \bar{B}$ is compact ( $B$ is relatively compact).

(b) $\alpha(B)=\alpha(\bar{B})$.

(c) $A \subset B \Rightarrow \alpha(A) \leq \alpha(B)$.

(d) $\alpha(A+B) \leq \alpha(A)+\alpha(B)$.

(e) $\alpha(c B)=|c| \alpha(B) ; \quad c \in \mathbb{R}$.

(f) $\alpha(\operatorname{conv} B)=\alpha(B)$.

For completeness we recall the definition of Caputo derivative of fractional order.

Definition 2.2. ([18]). The fractional (arbitrary) order integral of the function $h \in L^{1}([0, T], E)$ of order $r \in \mathbb{R}_{+}$is defined by

$$
I^{r} h(t)=\frac{1}{\Gamma(r)} \int_{0}^{t}(t-s)^{r-1} h(s) d s, \quad \text { for a.e. } t \in[0, T],
$$

where $\Gamma$ is the Euler gamma function defined by $\Gamma(r)=\int_{0}^{\infty} t^{r-1} e^{-t} d t, r>0$. 
Definition 2.3. ([18]). For a function $h \in A C^{n}(J, E)$, the Caputo fractional-order derivative of order $r$ of $h$ is defined by

$$
\left({ }^{c} D_{0}^{r} h\right)(t)=\frac{1}{\Gamma(n-r)} \int_{0}^{t}(t-s)^{n-r-1} h^{(n)}(s) d s, \quad \text { for a.e. } t \in[0, T],
$$

where $n=[r]+1$.

We need the following auxiliary lemmas $([18])$.

Lemma 2.4. Let $r>0$ and $h \in A C^{n}(J, E)$. Then the differential equation

$$
{ }^{c} D_{0}^{r} h(t)=0, \quad \text { for a.e. } t \in J
$$

has solutions $h(t)=c_{0}+c_{1} t+c_{2} t^{2}+\cdots+c_{n-1} t^{n-1}, c_{i} \in \mathbb{R}, i=0,1,2, \ldots, n-1$, $n=[r]+1$.

Lemma 2.5. Let $r>0$ and $h \in A C^{n}(J, E)$. Then

$$
I^{r c} D_{0}^{r} h(t)=h(t)+c_{0}+c_{1} t+c_{2} t^{2}+\cdots+c_{n-1} t^{n-1}, \quad \text { for a.e. } t \in J
$$

for some $c_{i} \in \mathbb{R}, i=0,1,2, \ldots, n-1, n=[r]+1$.

Definition 2.6. A map is said to be Carathéodory if

i $t \rightarrow f(t, u)$ is measurable for each $u \in E$.

ii $u \rightarrow F(t, u)$ is continuous for almost all $t \in J$.

For our purpose we will only need the following fixed point theorem, and the important Lemma.

Theorem 2.7. ([6, 20]) (Mönch's fixed point theorem). Let $D$ be a bounded, closed and convex subset of a Banach space such that $0 \in D$, and let $N$ be a continuous mapping of $D$ into itself. If the implication

$$
V=\overline{\operatorname{conv}} N(V) \text { or } V=N(V) \cup\{0\} \Rightarrow \alpha(V)=0
$$

holds for every subset $V$ of $D$, then Nhas a fixed point.

Lemma 2.8. ([15]) If $V \subset C(J ; E)$ is a bounded and equicontinuous set, then

(i) the function $t \rightarrow \alpha(V(t))$ is continuous on $J$, and

$$
\alpha_{c}(V)=\sup _{0 \leq t \leq T} \alpha(V(t)) .
$$

(ii) $\alpha\left(\int_{0}^{T} x(s) d s: x \in V\right) \leq \int_{0}^{T} \alpha(V(s)) d s$,

where

$$
V(s)=\{x(s): x \in V\}, s \in J .
$$




\section{Existence of Solutions}

First of all, we define what we mean by a solution of the IVP (1)-(3).

Definition 3.1. A function $y \in P C(J, E) \cap A C\left(J^{\prime}, E\right)$ is said to be a solution of (1)-(3) if $y$ satisfies $y(0)=y_{0},{ }^{c} D^{r} y(t)=f(t, y(t))$, for a.e. $t \in\left(s_{k}, t_{k+1}\right]$, and each $k=0, \ldots, m$, and $y(t)=g_{k}(t, y(t))$, for all $t \in\left(t_{k}, s_{k}\right]$, and every $k=1, \ldots, m$,

To prove the existence of solutions to (1)-(3), we need the following auxiliary lemmas.

Lemma 3.2. Let $0<r \leq 1$ and let $h: J \rightarrow E$ be integrable. Then linear problem

$$
\begin{gathered}
{ }^{c} D^{r} y(t)=h(t), \text { for each } t \in J_{k}:=\left(s_{k}, t_{k+1}\right], \quad k=0, \ldots, m, \\
y(t)=g_{k}(t), \text { for each } t \in J_{k}^{\prime}:=\left(t_{k}, s_{k}\right] \quad k=1, \ldots, m, \\
y(0)=y_{0}
\end{gathered}
$$

has a unique solution which is given by:

$$
y(t)= \begin{cases}y_{0}+\frac{1}{\Gamma(r)} \int_{0}^{t}(t-s)^{r-1} h(s) d s, & \text { if } t \in\left[0, t_{1}\right], \\ g_{k}(t), & \text { if } t \in J_{k}^{\prime} \quad k=1, \ldots, m, \\ g_{k}\left(s_{k}\right)+\frac{1}{\Gamma(r)} \int_{s_{k}}^{t}(t-s)^{r-1} h(s) d s, & \text { if } t \in J_{k} \quad k=1, \ldots, m .\end{cases}
$$

Proof. Assume that $y$ satisfies (4)-(6).

If $t \in\left[0, t_{1}\right]$ then

$$
{ }^{c} D^{r} y(t)=h(t) .
$$

Lemma 2.5 implies

$$
y(t)=y_{0}+\frac{1}{\Gamma(r)} \int_{0}^{t}(t-s)^{r-1} h(s) d s .
$$

If $t \in J_{1}^{\prime}=\left(t_{1}, s_{1}\right]$ we have $y(t)=g_{1}(t)$.

If $t \in J_{1}=\left(s_{1}, t_{2}\right]$, then Lemma 2.5 implies

$$
\begin{aligned}
y(t) & =y\left(s_{1}^{+}\right)+\frac{1}{\Gamma(r)} \int_{s_{1}}^{t}(t-s)^{r-1} h(s) d s \\
& =g_{1}\left(s_{1}\right)+\frac{1}{\Gamma(r)} \int_{s_{1}}^{t}(t-s)^{r-1} h(s) d s .
\end{aligned}
$$

If $t \in J_{2}^{\prime}=\left(t_{2}, s_{2}\right]$ we have $y(t)=g_{2}(t)$.

If $t \in J_{2}=\left(s_{2}, t_{3}\right]$ then again Lemma 2.5 implies

$$
\begin{aligned}
y(t) & =y\left(s_{2}^{+}\right)+\frac{1}{\Gamma(r)} \int_{s_{2}}^{t}(t-s)^{r-1} h(s) d s \\
& =g_{2}\left(s_{2}\right)+\frac{1}{\Gamma(r)} \int_{s_{2}}^{t}(t-s)^{r-1} h(s) d s .
\end{aligned}
$$


If $t \in J_{k}^{\prime}=\left(t_{k}, s_{k}\right]$ we have $y(t)=g_{k}(t)$.

If $t \in J_{k}=\left(s_{k}, t_{k+1}\right]$ then Lemma 2.5 implies

$$
\begin{aligned}
y(t) & =y\left(s_{k}^{+}\right)+\frac{1}{\Gamma(r)} \int_{s_{k}}^{t}(t-s)^{r-1} h(s) d s \\
& =g_{k}\left(s_{k}\right)+\frac{1}{\Gamma(r)} \int_{s_{k}}^{t}(t-s)^{r-1} h(s) d s .
\end{aligned}
$$

Conversely, assume that $y$ satisfies equation (7).

If $t \in\left[0, t_{1}\right]$, then $y(0)=y_{0}$ and, using the fact that ${ }^{c} D^{r}$ is the left inverse of $I^{r}$, we get

$$
{ }^{c} D^{r} y(t)=h(t) \text {, for each } t \in\left(0, t_{1}\right] .
$$

If $t \in J_{k}:=\left(s_{k}, t_{k+1}\right], \quad k=1, \ldots, m$, and using the fact that ${ }^{c} D^{r} C=0$, where $C$ is a constant, we get

$$
{ }^{c} D^{r} y(t)=h(t), \text { for each } t \in J_{k}:=\left(s_{k}, t_{k+1}\right], \quad k=1, \ldots, m .
$$

Also, we have easily that

$$
y(t)=g_{k}(t), \text { for each } t \in J_{k}^{\prime}:=\left(t_{k}, s_{k}\right], \quad k=1, \ldots, m .
$$

We are now in a position to state and prove our existence result for the problem (1)-(3) based on Mönch's fixed point. Let us list some conditions on the functions involved in the IVP (1)-(3).

(H1) The function $f: J \times E \rightarrow E$ satisfies the Carathéodory conditions.

(H2) There exists $p \in C\left(J, \mathbb{R}_{+}\right)$such that

$$
\|f(t, y)\| \leq p(t)\|y\| \text { for any } y \in E \text { and } t \in J
$$

(H3) $g_{k}$ are uniformly continuous functions and there exists $c_{k} \in C\left(J, \mathbb{R}_{+}\right)$such that

$$
\left\|g_{k}(t, y)\right\| \leq c_{k}(t)\|y\|, \text { for each } y \in E \text { and } t \in J, k=1, \ldots, m \text {. }
$$

(H4) For each bounded set $B \subset E$ we have

$$
\alpha\left(g_{k}(t, B)\right) \leq c_{k}(t) \alpha(B), t \in J
$$

(H5) For each bounded set $B \subset E$ we have

$$
\alpha(f(t, B)) \leq p(t) \alpha(B), t \in J .
$$

Let

$$
p^{*}=\sup _{t \in J} p(t), c^{*}=\max _{k=1, \ldots, m}\left(\sup _{t \in J}\left(c_{k}(t)\right)\right) .
$$


Theorem 3.3. Assume that assumptions (H1)-(H5) hold. If

$$
\frac{p^{*} T^{r}}{\Gamma(r+1)}+c^{*}<1
$$

then the IVP (1)-(3) has at least one solution $J$.

Proof. Transform the problem (1)-(3) into a fixed point problem. Consider the operator $N: P C(J, E) \rightarrow P C(J, E)$ defined by

$$
N(y)(t)= \begin{cases}y_{0}+\frac{1}{\Gamma(r)} \int_{0}^{t}(t-s)^{r-1} f(s, y(s)) d s, & \text { if } t \in\left[0, t_{1}\right], \\ g_{k}(t, y(t)), & \text { if } t \in J_{k}^{\prime}:=\left(t_{k}, s_{k}\right] \\ g_{k}\left(s_{k}, y\left(s_{k}\right)\right)+\frac{1}{\Gamma(r)} \int_{s_{k}}^{t}(t-s)^{r-1} f(s, y(s)) d s, & \text { if } t \in J_{k}:=\left(s_{k}, t_{k+1}\right]\end{cases}
$$

Clearly, the fixed points of operator $N$ are solutions of problem (1)-(3). Let

$$
r_{0} \geq \frac{\left\|y_{0}\right\|}{1-\frac{p^{*} T^{r}}{\Gamma(r+1)}-c^{*}}
$$

and consider the set

$$
D_{r_{0}}=\left\{y \in P C(J, E):\|y\|_{\infty} \leq r_{0}\right\}
$$

Clearly, the subset $D_{r_{0}}$ is closed, bounded and convex. We shall show that $N$ satisfies the assumptions of Theorem 2.7. The proof will be given in a couple of steps.

Step 1: $N$ is continuous.

Let $\left\{u_{n}\right\}$ be a sequence such that $u_{n} \rightarrow u$ in $P C(J, E)$. Then

for $t \in J_{k}$, we have

$$
\begin{aligned}
\left\|N\left(y_{n}\right)(t)-N(y)(t)\right\| & \leq\left\|g_{k}\left(t, y_{n}(t)\right)-g_{k}(t, y(t))\right\| \\
& +\frac{1}{\Gamma(r)} \int_{s_{k}}^{t}\left(t_{k}-s\right)^{r-1}\left\|f\left(s, y_{n}(s)\right)-f(s, y(s))\right\| d s,
\end{aligned}
$$

for $t \in\left[0, t_{1}\right]$, we have

$$
\left\|N\left(y_{n}\right)(t)-N(y)(t)\right\| \leq \frac{1}{\Gamma(r)} \int_{0}^{t}(t-s)^{r-1}\left\|f\left(s, y_{n}(s)\right)-f(s, y(s))\right\| d s
$$

and for $t \in J_{k}^{\prime}$, we have

$$
\left\|N\left(u_{n}\right)(t)-N(u)(t)\right\| \leq\left\|g_{k}\left(t, y_{n}(t)\right)-g_{k}(t, y(t))\right\| .
$$

Since $g_{k}$ is continuous and $f$ is of Carathéodory type, the Lebesgue dominated convergence theorem implies

$$
\left\|N\left(u_{n}\right)-N(u)\right\|_{\infty} \rightarrow 0 \text { as } n \rightarrow \infty .
$$


Consequently, $N$ is continuous.

Step 2: $N$ maps $D_{r_{0}}$ into itself.

For each $y \in D_{r_{0}}$, by (H2), (H3) and (10) we have for each $t \in J$,

$$
\begin{aligned}
\|N(y)(t)\| & \leq\left\|g_{k}(t, y(t))\right\|+\left\|y_{0}\right\|+\frac{1}{\Gamma(r)} \int_{0}^{t}(t-s)^{r-1}\|f(s, y(s))\| d s \\
& \leq c_{k}\|y(t)\|+\left\|y_{0}\right\|+\frac{1}{\Gamma(r)} \int_{s_{k}}^{t_{k+1}}(t-s)^{r-1} p(s)\|y(s)\| d s \\
& \leq\left\|y_{0}\right\|+r_{0}\left(\frac{p^{*} T^{r}}{\Gamma(r+1)}+c^{*}\right) \\
& \leq r_{0} .
\end{aligned}
$$

Step 3: $N\left(D_{r_{0}}\right)$ is bounded and equicontinuous.

By Step 2, it is obvious that $N\left(D_{r_{0}}\right) \subset P C(J, E)$ is bounded.

For the equicontinuous of $N\left(D_{r_{0}}\right)$, let $\tau_{1}, \tau_{2} \in J, \tau_{1}<\tau_{2}$ and $y \in D_{r_{0}}$. Then, for $\tau_{1}, \tau_{2} \in J_{k}$, we have

$$
\begin{aligned}
\left\|N(y)\left(\tau_{2}\right)-N(y)\left(\tau_{1}\right)\right\| & \left.=\frac{1}{\Gamma(r)} \int_{\tau_{1}}^{\tau_{2}}\left|\left(\tau_{2}-s\right)^{r-1}-\left(\tau_{1}-s\right)^{r-1}\right| \| f(s, y(s))\right) \| d s \\
& \leq 2 \frac{r_{0} p^{*}}{\Gamma(r+1)}\left[\tau_{2}^{r}-\tau_{1}^{r}\right]
\end{aligned}
$$

for $\tau_{1}, \tau_{2} \in\left[0, t_{1}\right]$, we have

$$
\begin{aligned}
\left\|N(y)\left(\tau_{2}\right)-N(y)\left(\tau_{1}\right)\right\| & =\frac{1}{\Gamma(r)} \int_{\tau_{1}}^{\tau_{2}}\left|\left(\tau_{2}-s\right)^{r-1}-\left(\tau_{1}-s\right)^{r-1}\right|\|f(s, y(s))\| d s . \\
& \leq 2 \frac{r_{0} p^{*}}{\Gamma(r+1)}\left[\tau_{2}^{r}-\tau_{1}^{r}\right]
\end{aligned}
$$

and for $\tau_{1}, \tau_{2} \in J_{k}^{\prime}$, we have

$$
\left\|N(y)\left(\tau_{2}\right)-N(y)\left(\tau_{1}\right)\right\|=\left\|g_{k}\left(\tau_{2}, y\left(\tau_{2}\right)\right)-g_{k}\left(\tau_{1}, y\left(\tau_{1}\right)\right)\right\| .
$$

As $\tau_{1} \rightarrow \tau_{2}$, the right-hand side of the above inequality tens to zero.

Now let $V$ be a subset of $D_{r_{0}}$ such that $V \subset \overline{c o n v}(N(V) \cup\{0\})$. Then $V$ is bounded and equicontinuous and therefore the function $t \rightarrow v(t)=\alpha(V(t))$ is continuous on $J$. By (H4), (H5), Lemma 2.8 and the properties of the measure $\alpha$ we have for each $t \in J$

$$
\begin{aligned}
v(t) & \leq \alpha(N(V)(t) \cup\{0\}) \\
& \leq \alpha(N(V)(t)) .
\end{aligned}
$$


If $t \in J_{k}$,

$$
\begin{aligned}
v(t) & \leq \alpha\left(g_{k}\left(s_{k}, V\left(s_{k}\right)\right)+\frac{1}{\Gamma(r)} \int_{s_{k}}^{t}(t-s)^{r-1} f(s, V(s)) d s\right) \\
& \leq c_{k}(t) \alpha(V(s))+\frac{1}{\Gamma(r)} \int_{s_{k}}^{t}(t-s)^{r-1} p(t) \alpha(V(s)) d s \\
& \leq c_{k}(t) v(s)+\frac{1}{\Gamma(r)} \int_{s_{k}}^{t}(t-s)^{r-1} p(t) v(s) d s \\
& \leq\|v\|_{\infty}\left(c^{*}+\frac{p^{*} T^{r}}{\Gamma(r+1)}\right)
\end{aligned}
$$

if $t \in\left[0, t_{1}\right]$

$$
\begin{aligned}
v(t) & \leq \alpha\left(\frac{1}{\Gamma(r)} \int_{0}^{t}(t-s)^{r-1} f(s, V(s)) d s\right) \\
& \leq \frac{1}{\Gamma(r)} \int_{0}^{t}(t-s)^{r-1} p(t) \alpha(V(s)) d s \\
& \leq \frac{1}{\Gamma(r)} \int_{0}^{t}(t-s)^{r-1} p(t) v(s) d s \\
& \leq\|v\|_{\infty}\left(\frac{p^{*} T^{r}}{\Gamma(r+1)}\right) \\
& \leq\|v\|_{\infty}\left(c^{*}+\frac{p^{*} T^{r}}{\Gamma(r+1)}\right),
\end{aligned}
$$

if $t \in J_{k}^{\prime}$

$$
\begin{aligned}
v(t) & \leq \alpha\left(g_{k}\left(s_{k}, V\left(s_{k}\right)\right)\right. \\
& \leq c_{k}(t) \alpha(V(s)) \\
& \leq c_{k}(t) v(s) \\
& \leq\|v\|_{\infty} c^{*} \\
& \leq\|v\|_{\infty}\left(c^{*}+\frac{p^{*} T^{r}}{\Gamma(r+1)}\right) .
\end{aligned}
$$

This means that

$$
\|v\|_{\infty}\left[1-\left(c^{*}+\frac{p^{*} T^{r}}{\Gamma(r+1)}\right)\right] \leq 0 .
$$

By (8) it follows that $\|v\|_{\infty}=0$; that is, $v(t)=0$ for each $t \in J$, and then $V(t)$ is relatively compact in $E$. In view of the Ascoli-Arzela theorem, $V$ is relatively compact in $D_{r_{0}}$. Applying now Theorem 2.7 we conclude that $N$ has a fixed point which is a solution of the problem (1)-(3). 


\section{An Example}

Let us consider the following infinite system of impulsive fractional initial value problem,

$$
\begin{gathered}
{ }^{c} D^{\frac{1}{2}} y_{n}(t)=\frac{1}{9+n+e^{t}} \ln \left(1+\left|y_{n}(t)\right|\right), \text { for a.e. } t \in\left(0, \frac{1}{3}\right] \cup\left(\frac{1}{2}, 1\right], \\
y_{n}(t)=\frac{1}{4+n+e^{t}} \sin \left|y_{n}(t)\right|, t \in\left(\frac{1}{3}, \frac{1}{2}\right], \\
y_{n}(0)=0 .
\end{gathered}
$$

Set

$$
E=l^{1}=\left\{y=\left(y_{1}, y_{2}, \ldots, y_{n}, \ldots,\right), \sum_{n=1}^{\infty}\left|y_{n}\right|<\infty\right\}
$$

$E$ is a Banach space with the norm

$$
\|y\|=\sum_{n=1}^{\infty}\left|y_{n}\right|
$$

Let

$$
\begin{gathered}
f(t, y)=\left(f_{1}(t, y), f_{2}(t, y), \ldots, f_{n}(t, y), \ldots\right), \\
f_{n}(t, y)=\frac{\ln \left(1+\left|y_{n}(t)\right|\right)}{9+n+e^{t}},
\end{gathered}
$$

and

$$
\begin{gathered}
g_{1}(t, y)=\left(g_{11}(t, y), g_{12}(t, y), \ldots, g_{1_{n}}(t, y), \ldots\right), \\
g_{1_{n}}(t, y)=\frac{\sin \left|y_{n}(t)\right|}{4+n+e^{t}}
\end{gathered}
$$

Clearly conditions (H2) and (H3) hold with

$$
p(t)=\frac{1}{9+e^{t}}, \text { and } c_{1}(t)=\frac{1}{4+e^{t}} .
$$

We shall check that condition (8) is satisfied with $r=\frac{1}{2}, T=1, P^{*}=\frac{1}{10}$ and $c^{*}=\frac{1}{5}$. Indeed

$$
\left(\frac{p^{*} T^{r}}{\Gamma(r+1)}+c^{*}\right)=\frac{1}{5 \sqrt{\pi}}+\frac{1}{5}<1 .
$$

Then by Theorem 3.3 the problem (11)-(13) has at least one solution.

\section{Acknowledgement}

The authors are grateful to the referee for the helpful remarks. 


\section{References}

[1] S. Abbas, M. Benchohra, Uniqueness and Ulam stabilities results for partial fractional differential equations with not instantaneous impulses, Appl. Math. Comput. 257 (2015) 190-198.

[2] S. Abbas, M. Benchohra, M.A. Darwish, New stability results for partial fractional differential inclusions with not instantaneous impulses, Frac. Calc. Appl. Anal. 18 (1) (2015) 172-191.

[3] S. Abbas, M. Benchohra, G.M. N'Guérékata, Topics in Fractional Differential Equations, Springer-Verlag, New York, 2012.

[4] S. Abbas, M. Benchohra, G.M. N'Guérékata, Advanced Fractional Differential and Integral Equations, Nova Science Publishers, New York, 2015.

[5] R.P. Agarwal, S. Hristova, D. O'Regan, Non-Instantaneous Impulses in Differential Equations, Springer, New York, 2017.

[6] R.P. Agarwal, M. Meehan, D. O'Regan, Fixed Point Theory and Applications, Cambridge University Press, Cambridge, 2001.

[7] R.R. Akhmerov, M.I. Kamenskii, A.S. Patapov, A.E. Rodkina, B.N. Sadovskii, Measures of Noncompactness and Condensing Operators, trans. from the Russian by A. Iacob, Birkhäuser Verlag, Basel, 1992.

[8] J.C. Alvàrez, Measure of noncompactness and fixed points of nonexpansive condensing mappings in locally convex spaces, Rev. Real. Acad. Cienc. Exact. Fis. Natur. Madrid 79 (1985) 53-66.

[9] J. Banaś, K. Goebel, Measures of Noncompactness in Banach Spaces, Marcel Dekker, New York, 1980.

[10] J. Banaś, B. Rzepka, An application of a measure of noncompactness in the study of asymptotic stability, Appl. Math. Lett. 16 (2003) 1-6.

[11] J. Banaś, K. Sadarangani, On some measures of noncompactness in the space of continuous functions, Nonlinear Anal. 68 (2008) 377-383.

[12] D.D. Bainov, P.S. Simeonov, Systems with Impulse Effect, Horwood, Chichester, 1989.

[13] M. Benchohra, J. Henderson, S.K. Ntouyas, Impulsive Differential Equations and Inclusions, Hindawi Publishing Corporation, Vol 2, New York, 2006.

[14] M. Benchohra, J. Henderson, D. Seba, Measure of noncompactness and fractional differential equations in Banach spaces, Commun. Appl. Anal. 12 (4) (2008) 419428. 
[15] D. Guo, V. Lakshmikantham, X. Liu, Nonlinear Integral Equations in Abstract Spaces, Kluwer Academic Publishers Group, Dordrecht, 1996.

[16] E. Hernández, D. O'Regan, On a new class of abstract impulsive differential equations, Proc. Amer. Math. Soc. 141 (2013) 1641-1649.

[17] R. Hilfer, Applications of Fractional Calculus in Physics, World Scientific, Singapore, 2000.

[18] A.A. Kilbas, H.M. Srivastava, J.J. Trujillo, Theory and Applications of Fractional Differential Equations, Elsevier Science B.V., Amsterdam, 2006.

[19] V. Lakshmikantham, D.D. Bainov, P.S. Simeonov, Theory of Impulsive Differential Equations, Worlds Scientific, Singapore, 1989.

[20] H. Mönch, Boundary value problems for nonlinear ordinary differential equations of second order in Banach spaces, Nonlinear Anal. 4 (1980) 985-999.

[21] H. Mönch, G.F. Von Harten, On the Cauchy problem for ordinary differential equations in Banach spaces, Archiv. Math. Basel 39 (1982) 153-160.

[22] M. Pierri, D. O'Regan, V. Rolnik, Existence of solutions for semi-linear abstract differential equations with not instantaneous impulses, Appl. Math. Comput. 219 (2013) 6743-6749.

[23] I. Podlubny, Fractional Differential Equations, Academic Press, San Diego, 1999.

[24] A.M. Samoilenko, N.A. Perestyuk, Impulsive Differential Equations, World Scientific, Singapore, 1995.

[25] Y. Zhou, Basic Theory of Fractional Differential Equations, World Scientific, Singapore, 2014.

\section{DOI: $10.7862 / \mathrm{rf} .2018 .4$}

\section{Mouffak Benchohra}

email: benchohra@yahoo.com

ORCID: 0000-0003-3063-9449

Laboratory of Mathematics

Djillali Liabes University of Sidi-Bel-Abbès

P.O. Box 89, Sidi Bel-Abbès 22000

ALGERIA 


\section{Mehdi Slimane}

email: m1slim@yahoo.com

ORCID: 0000-0002-1556-3709

Laboratory of Mathematics

Djillali Liabes University of Sidi-Bel-Abbès

P.O. Box 89, Sidi Bel-Abbès 22000

ALGERIA

Received 28.12.2017

Accepted 23.04.2018 